\title{
SELECCIÓN DE BUENAS PRÁCTICAS PARA LA INCLUSIÓN SOCIAL EN EL MARCO DE UN PROYECTO EUROPEO:URBAL-10 ${ }^{1}$
}

\author{
REMEdios LaRrubia VARgas \\ SUSANA R. NAVARRo RODRÍGUEZ
}

\begin{abstract}
RESUMEN
La constatación del protagonismo y relevancia que las actuaciones en torno a la "exclusión social" están cobrando dentro del ámbito municipal, así como los cambios en el planteamiento de las acciones a desarrollar y en sus objetivos, ha llevado a buscar un instrumento que permita ahondar en la calidad de las acciones que desarrollan las Administraciones Públicas. En esta línea el Área de Bienestar Social del Ayuntamiento de Málaga en el marco de un Proyecto Europeo en el que colabora con distintos socios iberoamericanos, ha llevado a cabo la elaboración, selección y divulgación de "Buenas Prácticas" con un claro fin demostrativo. Es este proceso de selección y el marco teórico donde se inserta las Buenas Prácticas el objetivo del presente trabajo.
\end{abstract}

\begin{abstract}
The verification of the protagonism and relevance that the performances around the social exclusion are getting paid inside the municipal environment, as well as the changes in the position of the actions to develop and in their objectives, it has taken to look for an instrument that allows to deepen in the quality of the actions that you/they develop the Public
\end{abstract}

1. Este artículo forma parte de un trabajo realizado para el área de bienestar social del Ayuntamiento de Málaga, dentro del marco del Proyecto Urbal-10, proyecto aprobado y subvencionado por la Comisión Europea. El proyecto ha contado con los siguientes socios: Aserrí de Costa Rica, Feira de Santana de Brasil, Lima y Oyon de Perú, Navolato de Méjico, El Bosque de Chile, I.M.F.E. de Granada, España y F.I.D.D.E.M (con sede en St. Denis, Francia) bajo la coordinación del Ayuntamiento de Málaga. Uno de los objetivos del Proyeto Urbal es promover el intercambio de experiencias y difusión de Buenas Prácticas en la elaboración de instrumentos de planificación y evaluación de políticas sociales mediante el uso de un sistema de información geográfica que permita el análisis de indicadores sociales y su aplicación a un territorio para lograr la "Inclusión Social". 
Administrations. In this line the Area of Social Well-being of the City council of Málaga in the mark of an European Project in which collaborates with different Ibero-American partners, has carried out the elaboration, selection and popularization of "Good Practical" with the purpose of that have a demonstrative character. It is this selection process and the theoretical mark where it is inserted the Good ones Practical the objective of the present work.

\section{INTRODUCCIÓN}

El desarrollo de las nuevas tecnologías y la globalización de la economía y de la información imponen mutaciones profundas en el mundo actual. Dichas mutaciones alteran las situaciones económicas y sociales en el ámbito local e implican nuevas necesidades de la población y de las empresas que van más allá de las necesidades de los servicios básicos que generalmente esperamos que presten las instituciones públicas.

Estas nuevas circunstancias conducen a un replanteamiento de la noción de servicio público. Lo que se cuestiona, aún más que la apertura de nuevos ámbitos de intervención, son los métodos de apoyo tradicionalmente utilizados. Actualmente, parece cada vez más inadecuada la organización sectorializada y normalizada de la ayuda pública. Las nuevas necesidades que debe satisfacer el servicio público suponen la puesta en funcionamiento de soluciones específicas, pensadas localmente y que superen las barreras tradicionales entre sectores. Todo esto implica la aparición de nuevas prácticas del servicio público, que son más difíciles de establecer en la medida en que representan una ruptura cultural profunda con la idea que tradicionalmente tenemos del mismo. Los entes locales han sido los primeros afectados por esta formidable transformación, pues son los principales representantes de los servicios públicos al nivel de la población. Estas nuevas técnicas llevadas a cabo por los servicios públicos han supuesto un cambio cualitativo en su forma de actuar, de forma que los Ayuntamientos han pasado de preocuparse de manera primordial por la protección a incluir entre sus acciones planes que, de manera integral, atiendan sus necesidades, es decir, las personas con problemáticas sociales han pasado de ser consideradas sujeto de intervención a ser consideradas protagonistas de su propia vida y agente de cambio.

\section{LA GESTIÓN DE LA CALIDAD EN LA PRESTACIÓN DE LOS SERVICIOS PÚBLICOS: LOS MODELOS DE CALIDAD}

En el área de los Servicios Sociales la mejora de la calidad de servicio ha sido una cuestión que ha tenido un especial desarrollo en las últimas décadas, lo que ha llevado a la necesidad de asumir nuevas prácticas que mejoren la 
calidad de los servicios sociales y que han generado la crisis y el cuestionamiento de las prácticas tradicionales.

La definición sobre calidad del servicio es una cuestión que todavía no está cerrada, existen muchos y variados acercamientos al tema, así como diferencias en torno a su mayor o menor dificultad de definición respecto a las empresas industriales. A pesar de la diversidad de opiniones, en el conjunto de definiciones que la literatura ofrece parece que hay una serie de hallazgos con cierto consenso, de forma que la calidad de un servicio es entendida en relación a la satisfacción de las expectativas de las personas-clientes, lo que supone en la práctica cuidar de manera fundamental y continua esta interacción. Esto es así en la medida que las expectativas de las personas no son estables, sino que se definen en un proceso en función de las circunstancias y en un proceso de negociación social.

Para la Gestión de esta Calidad se han propuesto diversos modelos de calidad. Los modelos que con más frecuencia se están implementando en el área de los servicios sociales son los cuatro que aparecen recogidos en el cuadro 1. Cada uno de estos modelos posee una finalidad, tiene unas ventajas y unos inconvenientes, si bien todos ellos cumplen el objetivo final que es la mejora y la adaptación de los servicios públicos a las nuevas tendencias. De esta manera cada órgano de la Administración elegirá aquél modelo que mejor responda a sus expectativas.

En relación a la gestión de la "Calidad de los Servicios" algunos autores, entre ellos Bradley (1990), consideran que es el modelo de "Buenas Prácticas o Códigos de Conducta" el que mejor se adapta a los Servicios de Bienestar. Este autor propone un modelo de calidad para la prestación de servicios bastante consensuado. Como punto de partida define la calidad en "términos de logros o resultados" que consiguen las personas. 


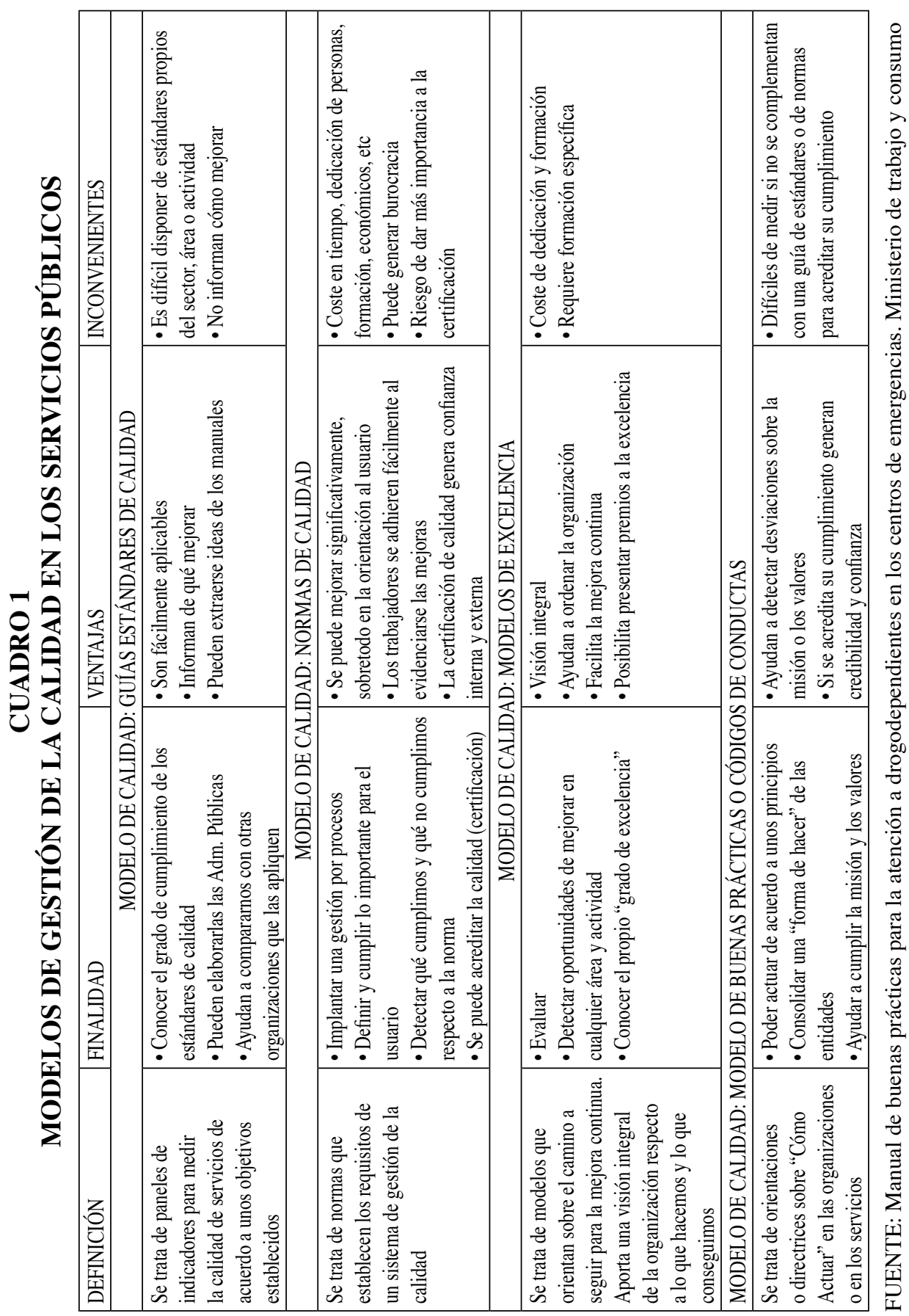




\section{EL MODELO DE BUENAS PRÁCTICAS}

De entre los modelos expuestos para implementar la Gestión de calidad en torno a la Inclusión social, el Proyecto Urbal-10 ha optado por el de "Buenas Prácticas" y la elaboración de un manual que suponga la presentación de una serie de orientaciones o directrices sobre "Cómo Actuar" en la organización y gestión de los servicios sociales, con el objetivo de superar la exclusión social.

Son múltiples y variadas las definiciones existentes sobre lo que son las Buenas Prácticas, no obstante del análisis del conjunto de definiciones se puede extraer los siguientes elementos comunes que sirven para conformar el concepto:

- Una acción o conjunto de acciones, valores y principios en un campo concreto y con un claro objetivo expreso: establecer metas, patrones, normas.

- Repercusiones tangibles y mensurables: satisfacer las necesidades y expectativas de los clientes.

- Mejora evidente de la situación de partida que era insatisfactoria.

- Servir de modelo para otros territorios. Ser el punto de partida y nunca de llegada

El área de Bienestar Social del Ayuntamiento de Málaga para el propósito que le ocupa ha considerado como buena práctica a aquella forma continuada de hacer que introduce mejoras en los procesos y actividades que tienen lugar en cualquier organización, mejoras orientadas a producir resultados positivos sobre la calidad de vida de las personas expuestas a situaciones de vulnerabilidad social. De este modo, la calidad puede entenderse como un movimiento que persigue la mejora continuada del servicio que se presta, nunca su punto de llegada.

Para la selección de buenas prácticas hay que considerar cuatro aspectos fundamentales:

1. Elegir y precisar los criterios de selección de las Buenas Prácticas

2. Estudiar las Buenas Prácticas adecuadas en relación con dichos criterios

3. Verificar la pertinencia de las Buenas Prácticas detectadas

4. Divulgación de las Buenas Prácticas seleccionadas

Para cumplir estas fases el paso previo es rellenar por parte de cada participante una "Ficha Identificativa" de cada actividad que ellos consideren una 
Buena Práctica. Las fichas suelen ser variadas, si bien la mayoría de ellas recogen unos aspectos comunes que son los que aparecen en el cuadro 2 y han sido los utilizados por el Ayuntamiento de Málaga en su proceso de selección.

\section{FICHA PARA LA ELABORACIÓN DE BUENAS PRÁCTICAS}

\begin{tabular}{|c|c|}
\hline ASPECTOS GENERALES & ASPECTOS ESPECÍFICOS \\
\hline 1.- DATOS GENERALES & $\begin{array}{l}\text { Se detalla y se exponen los datos básicos de la } \\
\text { experiencia, los datos de identificación: } \\
\text { - Título de la experiencia } \\
\text { - Municipio en el que se desarrolla } \\
\text { - Institución o instituciones implicadas } \\
\text { - Edades a las que va dirigida la experiencia } \\
\text { - Problemática de las personas a la que se dirige la } \\
\text { experiencia } \\
\text { - Duración de la experiencia } \\
\text { - Valoración de resultados: Valoración de la utilidad de } \\
\text { la práctica, desde la perspectiva del socio que ha hecho } \\
\text { la propuesta, con indicación de las posibilidades de } \\
\text { imitación, en términos de medios o de costes }\end{array}$ \\
\hline $\begin{array}{l}\text { 2.-DESARROLLO DE LA } \\
\text { EXPERIENCIA }\end{array}$ & $\begin{array}{l}\text { 1.- Se realizará un resumen de la experiencia, atendiendo } \\
\text { a : } \\
\text { - Situación anterior al desarrollo de la experiencia } \\
\text { - Actuación } \\
\text { - Situación posterior al desarrollo de la actuación } \\
\text { 2.- Se expondrán los objetivos generales y específicos } \\
\text { 3.- Contenidos: temas-clave de la experiencia } \\
\text { 4.- Metodología } \\
\text { 5.- Contexto de aplicación }\end{array}$ \\
\hline 3.-PERSONA DE CONTACTO & $\begin{array}{l}\text { El carácter demostrativo o revelador supone asegurarse } \\
\text { de la pertinencia de la Buena Práctica, una herramienta } \\
\text { importante la constituyen las informaciones recogidas } \\
\text { de los expertos que trabajan sobre un tema específico. } \\
\text { Una breve entrevista con los responsables del proyecto } \\
\text { confirmará si la acción corresponde a los documentos } \\
\text { consultados para su detección. }\end{array}$ \\
\hline
\end{tabular}

El paso posterior es la selección de las Buenas Prácticas conforme a unos criterios preestablecidos. Los criterios utilizados por el Área de Bienestar Social del Ayuntamiento de Málaga se han basado en los llevados a cabo por la Comunidad Internacional, en concreto en su segunda convocatoria del concurso de Buenas Prácticas del Comité Hábitat II (1996) sobre asentamientos humanos desarrollados en la Segunda Conferencia de Naciones Unidas, ce- 
lebrada en Estambul (Turquía, 1996). Este organismo ha considerado que para que una actuación merezca el calificativo de Buena Práctica debe reunir 7 requisitos, no todos siempre de estricto cumplimiento, recogidos en el cuadro 3.

\section{CUADRO 3 \\ CRITERIOS DE SELECCIÓN DE BUENAS PRÁCTICAS}

\begin{tabular}{|c|c|}
\hline CRITERIOS GENERALES & CRITERIOS ESPECÍFICOS \\
\hline IMPACTO & $\begin{array}{l}\text { - Demostrar mejoras tangibles en las condiciones de vida de } \\
\text { las personas en cualquiera de las áreas temáticas propuestas }\end{array}$ \\
\hline ASOCIACIÓN & $\begin{array}{l}\text { - Asociación entre al menos dos entidades: (Administración,. } \\
\text { ONG’s, Sector privado y fundaciones, Organismos } \\
\text { Internacionales, Centros académicos y/o de investigación, } \\
\text { Asociaciones profesionales, medios de comunicación, líderes } \\
\text { cívicos, voluntarios) }\end{array}$ \\
\hline SOSTENIBILIDAD & $\begin{array}{l}\text { Esta se manifiesta en cambios duraderos en : } \\
\text { - Marcos legislativos, normas, ordenanzas y estándares. } \\
\text { - Políticas sociales y estrategias con posibilidades de } \\
\text { aplicación en otras situaciones. } \\
\text { - Marcos institucionales y procesos para la adopción de } \\
\text { decisiones. } \\
\text { - Sistema de administración y gestión eficientes, transparentes } \\
\text { y responsables. } \\
\text { - Sostenibilidad económica }\end{array}$ \\
\hline $\begin{array}{l}\text { PERTINENCIA Y } \\
\text { DESARROLLO DE } \\
\text { COMPETENCIAS }\end{array}$ & $\begin{array}{l}\text { - Experiencia coherente con los objetivos de partida } \\
\text { - Desarrollo de competencia en diferentes ámbitos: } \\
\text { comunicación, búsqueda de información, solución de } \\
\text { problemas, aprendizajes, etc. }\end{array}$ \\
\hline $\begin{array}{l}\text { LIDERAZGO Y } \\
\text { FORTALECIMIENTO DE } \\
\text { LA COMUNIDAD }\end{array}$ & $\begin{array}{l}\text { - Se trata de iniciativas que inspiren actividades innovadoras, } \\
\text { fomenten cambios. } \\
\text { - Fortalecimiento de la participación de los vecinos, } \\
\text { asociaciones, comunidades, etc. } \\
\text { - Posibilidad de intercambio, transferencia y aplicación de } \\
\text { experiencias. } \\
\text { - Medios adecuados a las condiciones locales. }\end{array}$ \\
\hline
\end{tabular}

Una vez identificados los criterios de elección de las Buenas Prácticas el siguiente paso es encontrar y seleccionar buenos "Ejemplos de Buenas Prácticas" que correspondan a los criterios marcados y su posterior divulgación mediante un catálogo o manual. 


\section{BUENAS PRÁCTICAS PARA LA INCLUSION SOCIAL}

El área de Bienestar Social del Ayuntamiento de Málaga, dentro del marco de un Proyecto europeo y en líneas con las nuevas tendencias y técnicas presentes en los servicios sociales, ha puesto en práctica una de estas técnicas en aras a la mejora de los servicios públicos: las Buenas Prácticas. En relación lógica con los objetivos del Proyecto Urbal-10, las Buenas Prácticas que se solicitaron y se seleccionaron como punto de partida tendría que tener como temática principal la lucha contra la exclusión social y como metodología básica el sistema de indicadores sociales y su posible implementación en un SIG (Sistema de Información Geográfica). Con posterioridad, desde el punto de vista metodológico se hizo una pequeña concesión en la línea que todos los proyectos desarrollados para el diagnóstico social deberían considerarse positivamente ya que el marco lógico de intervención también delimita como objetivo específico propiciar instrumentos de diagnóstico útil que permitan mejorar el diseño de políticas sociales, aunque en principio no estén integrados en un sistema de cartografía digital.

En virtud de lo anterior las prácticas seleccionadas podían ser de dos tipos, en primer lugar, aquellas que se adecuen tanto en temática (problemas sociales), metodología (indicadores sociales) como en técnicas (uso de los SIG), en segundo lugar, aquellas que se adecuen sólo temática y metodológicamente a los objetivos del proyecto, siendo su reto inmediato la implementación de un SIG.

Sin embargo, desde este punto de vista las políticas sociales para la superación de la exclusión social no serían una Buena Práctica, sino el objetivo final de las Buenas Prácticas que vamos a identificar.

El objetivo general es reconocer y estimular las experiencias que se consideran exitosas en ara a la erradicación de la exclusión social, y que reúnan los elementos esenciales de una Buena Práctica municipal, participativa, de alto impacto, que promueva la asociatividad institucional, demuestre una adecuada sostenibilidad y sea pertinente, coherente y eficiente con los recursos utilizados y que se corresponden con los criterios de evaluación especificados en el cuadro 3.

Los objetivos específicos han sido:

- Estimular y reconocer Buenas Prácticas en torno a la exclusión-vulnerabilidad social.

- Registrar y sistematizar prácticas municipales destacadas potenciando su contribución a la gestión de los mecanismos a favor de la erradicación de la exclusión social.

- Difundir el conocimiento de las experiencias de los diferentes municipios participantes en el proyecto Urbal-10, facilitando el contacto entre instituciones y experiencias. 
El proceso de selección de "Buenas Prácticas" ha tenido dos fases. Una primera fase en relación al seminario realizado en Málaga en el mes de septiembre de 2005 para el que los socios hicieron llegar un glosario de lo que ellos consideraban Buenas Prácticas. Todas ellas fueron evaluadas y señaladas sus principales carencias a fin de que fueran corregidas. Una segunda fase, definitiva, en la que se depuró la selección de las prácticas a fin de elegir aquellas que se consideraban más pertinentes con los objetivos del proyecto.

En la primera fase fueron enviadas un total de 27 prácticas, 19 de las cuales habían finalizado o estaban en proceso de desarrollo, ocho de ellas, pertenecientes al municipio de Aserrí (Costa Rica) estaban pendientes de implantación. La distribución por países de las buenas prácticas enviadas en aquel momento ponía de manifiesto que por su número el socio que se situaba en primera posición era el municipio de Feira de Santana que presentaba las 2/3 parte de las prácticas, seguido por Aserrí (11\%), Granada y Navolato cada uno con dos prácticas, lo que suponía en torno a $7.5 \%$ de las prácticas presentadas. En este momento los municipios de Málaga y Lima optaron por no presentar práctica alguna.

En relación a la temática específica y concreta a la que iban dirigida las Prácticas sólo tres de ellas presentaban una alta pertinencia temática, metodológica y técnica con los objetivos específicos de esta parte del Proyecto URBAL. Especial mención hay que hacer a las prácticas que fueron presentadas por Granada (España) y El Bosque (Chile) y que podían ser clasificadas como prácticas tipos. La práctica enviada por el municipio de Oyon (Perú), metodológicamente respondía a la cartografía digital, auque con el inconveniente de ser un proyecto nacional y no a nivel municipal lo que era objetivo prioritario.

En cuanto al resto de las prácticas presentadas ninguna de ellas utilizaban la cartografía digital, los Sistemas de Información Geográficos, compresible, desde el momento en que el proyecto Urbal tiene como una de sus metas la implantación de esta metodología.

Desde el punto de vista metodológico la mayor parte de las prácticas tenían cabida en el proyecto, aunque hay que puntualizar que no todas utilizaban explícitamente indicadores sociales. No obstante como el planteamiento y realización de un proyecto conlleva una fase previa de diagnóstico social, pensamos que podíamos considerar como pertinente incluirlas en el proyecto, al ser un paso indiscutible y previo al diseño de políticas sociales.

Por su temática de análisis algunos proyectos eran difíciles de ser incluidos dentro del ámbito de la Vulnerabilidad/Exclusión social, puesto que no suponían un cambio, una mejora de las condiciones de vida ciudadana, eran el caso de las prácticas remitidas por Navolato y Aserrí, referentes a "simulación de escenarios de intensidad símica, o la jerarquización de la red vial”. 
Además la mayoría de las prácticas presentaban un serie de carencias en cuanto a su presentación y formalización al no adaptarse a los requisitos que se recogían en la ficha identificativa. En general, las carencias principales que se detectaron fueron un vago resumen de la experiencia (un resumen pormenorizado es esencial en cuanto que la experiencia debe tener un efecto demostrativo y poder ser puesta en práctica en otros municipios); escasa valoración de los resultados, especialmente en cuanto a las posibilidades de transferencia de la práctica, su coste, financiación y medios necesarios, el impacto y la durabilidad de la misma (aspectos fundamentales de cara a la transferencia a otras zonas); identificación de las asociaciones e instituciones implicadas (no en vano para que un proyecto pueda ser considerado buena práctica se necesita la intervención de al menos dos instituciones).

La conclusión fundamental de esta primera fase fue la toma de conciencia de las carencias que presentaban las prácticas enviadas y el compromiso de hacer una selección de prácticas más adecuadas.

La segunda fase de selección de buenas prácticas se inicia lógicamente una vez finalizado el seminario, cuando los diferentes socios desde sus respectivos municipios realizaron un nuevo envío de "Buenas Prácticas". La mayoría de las prácticas que con anterioridad se habían considerado válidas fueron nuevamente enviadas perfectamente formalizadas de acuerdo con los ítems de la ficha identificativa; los miembros restantes remitieron prácticas distintas formalmente correctas, de las que se seleccionaron aquellas que técnica y metodológicamente correspondían con los objetivos del proyecto y serán a las que se dé divulgación mediante un Manual de Buenas Prácticas.

\subsection{Prácticas Definitivas Seleccionadas}

Como se aprecia en el cuadro 4 las Buenas Prácticas seleccionadas definitivamente han sido 13, de ellas, cinco (dos de Granada, dos de Chile y una de Oyon) fueron enviadas y seleccionadas desde el principio, las ocho restantes han sido totalmente nuevas. Han sido las prácticas remitidas por Granada (especialmente la denominada "Atlas de empleo y atlas de formación profesional ocupacional de Granada Capital"), las de la municipalidad de El Bosque y las de Málaga, las que se adecuan con mayor pertinencia a los objetivos del proyecto tanto temática, metodológica y técnicamente, esto es, se basan en un sistema de indicadores sociales y se implementan a través de un SIG con el objetivo de hacer frente a situaciones de vulnerabilidad social. La práctica de Oyon también sigue estas pautas aunque su escala territorial de análisis es más pequeña.

Las prácticas restantes se adecuan perfectamente a la temática y a la metodología propuestas por el proyecto, siendo su objetivo futuro la adaptación técnica, es decir su materialización en un SIG. 
En lógica relación con los criterios de selección de Buenas Prácticas, las experiencias seleccionadas cumplen la mayor parte de los criterios de selección establecidos, aunque no todas en el mismo grado.

El criterio de impacto es aplicable a todas ellas en cuanto que a corto o largo plazo han supuesto o van a suponer una mejora en diferentes aspectos de la vida social de las poblaciones donde se aplica la experiencia.

El criterio de participación también ha sido muy valorado en todas las prácticas, por lo general en la mayoría de ellas han participado más de dos instituciones en su desarrollo, a lo que hay que sumar en algunas prácticas la intervención de empresas privadas, ONGs, particulares, etc.

También las prácticas han sido muy pertinentes en cuanto al criterio de sostenibilidad, puesto que la mayor parte de ellas suponen una continuidad en el tiempo y su renovación paulatina, además los costes económicos son bastantes asumibles, de forma que la relación coste/impacto es muy ajustada, siendo pues viable su transportabilidad a otros espacios. Además también hay que considerar muy positivamente que la mayoría de las actuaciones han contado con organizaciones sociales que colaboran en todo el proceso.

Resulta también muy positivo el que la mayoría de las prácticas concentren sus actuaciones en aras a potenciar la conciencia comunitaria puesto que a través de sus organizaciones sociales y económicas fortalecen la cohesión social, la integración cultural y la tolerancia como principal capital que evite fracturas sociales importantes.

Por último se trata de acciones pioneras y con carácter demostrativo.

\section{CUADRO 4 \\ DISTRIBUCIÓN DE LAS BUENAS PRÁCTICAS SELECCIONADAS}

\begin{tabular}{|l|c|l|}
\hline PAÍS & N $^{\text {PRACTICAS }}$ & DENOMINACIÓN PRÁCTICAS \\
\hline ASERRI (COSTA RICA) & 1 & $\begin{array}{l}\text { ・Análisis de la Situación Integral de } \\
\text { Salud del Cantón de Aserrí }\end{array}$ \\
\hline FEIRA DE SANTANA (BRASIL) & 1 & • Niños fuera de la calle \\
\hline OYON (PERÚ) & 1 & • Cartografía nacional \\
\hline NAVOLATO (MÉJICO) & 3 & $\begin{array}{l}\text { - Prevención de las adicciones a los } \\
\text { jóvenes } \\
\bullet \text { Intervención psicosocial y } \\
\text { comunitaria con adolescentes y } \\
\text { jóvenes } \\
\bullet \text { Sensibilización comunitaria } \\
\text { "Violencia Intrafamiliar". }\end{array}$ \\
\hline
\end{tabular}




\begin{tabular}{|l|c|l|}
\hline PAÍS & No PRACTICAS & DENOMINACIÓN PRÁCTICAS \\
\hline EL BOSQUE (CHILE) & 2 & $\begin{array}{l}\text { ・ Un SIG como instrumento de } \\
\text { planificación social-territorial. Una } \\
\text { experiencia: “Campaña matrícula de } \\
\text { educación 2006-SIGECUM". } \\
\text { - Un SIG aplicado a la Gestión } \\
\text { Municipal }\end{array}$ \\
\hline GRANADA (ESPAÑA) & 2 & $\begin{array}{l}\text { - Atlas de empleo y atlas de } \\
\text { formación profesional ocupacional } \\
\text { de Granada capital } \\
\bullet \text { Modelo sociodemográfico: Atlas } \\
\text { social de la ciudad de Alicante }\end{array}$ \\
\hline MALAGA (ESPAÑ) & 2 & $\begin{array}{l}\text { - Diseño y ejecución del diagnóstico } \\
\text { de las Zonas de Trabajo Social del } \\
\text { municipio de Málaga } \\
\text { - Estudio socio-territorial de la } \\
\text { ciudad de Málaga }\end{array}$ \\
\hline LIMA (PERÚ) & 1 & $\begin{array}{l}\text { • Programa de intervención para el } \\
\text { desarrollo solidario de comunidades } \\
\text { (PIDESC). }\end{array}$ \\
\hline TOTAL & 13 & \\
\hline
\end{tabular}

\subsection{Características básicas y generales de las propuestas seleccionadas}

Uno de los aspectos que hay que considerar de las propuestas presentadas y seleccionadas, dentro del objetivo de la exclusión social, es la diversidad temática. Teniendo en cuenta aquellas propuestas seleccionadas y tras su evaluación para su inclusión en el manual divulgativo, deben destacarse los siguientes aspectos sobre los que focalizan su atención:

- Tienen en común, en primer lugar, la consideración conjunta de los aspectos territoriales y sociales de la exclusión social. Para el diagnóstico de situaciones vulnerables o de riesgos se tienen en cuenta la actuación sobre parámetros sociales de exclusión en un escenario físico muy concreto (un barrio, cantón, comunidad) especialmente destaca en este sentido las prácticas de Navolato (Méjico) que actúan sobre polígonos y colonias concretas, las de Málaga (define las Zonas de Trabajo Social) y la de El Bosque (se centra en un territorio muy definido, "La Comuna").

- En segundo lugar, se maneja explícitamente (aquellas que ya han concluido) o implícitamente (aquellas que están en curso y a punto de finalizar), la idea de una mejora de las condiciones sociales mediante cualquiera de los programas de actuación. 
- En tercer lugar, los programas centrados en la mejora social, focalizan su atención en el desarrollo comunitario y la integración social y laboral, centrando sus actividades en un colectivo específico, altamente vulnerable (jóvenes, adolescentes y mujeres). En esta línea son destacables los programas enviados por Navolato (Méjico) y Granada (España).

- También, es de destacar, pues es un punto importante en la evaluación de la práctica, el interés y el esfuerzo por el establecimiento de contactos y elaboración conjunta por parte de diversos agentes sociales, públicos y privados, aunque predominen la capacidad de propuesta hecha efectiva por parte de las instituciones locales (ayuntamientos, concejalías, asociaciones locales, ONGs, empresas privadas, particulares, etc).

- En el conjunto de actuaciones el organismo que posee el liderazgo es el municipal con los Ayuntamientos y organismos institucionales en cabeza, si bien tienen una presencia destacable la sociedad civil o determinados colectivos que se organizan para el desarrollo de iniciativas dirigidas a solucionar distintas problemáticas: ONGs, Asociaciones, empresas mixtas. Lo que puede interpretarse como un signo de avance en la concienciación de la necesidad de trabajar de forma asociada, a la vez que de la comprensión y la complejidad de los problemas sociales que exigen para su tratamiento una alta cooperación.

- Son significativas las prácticas dirigidas a los problemas de integración y equidad social que han pasado a ser un reto fundamental para mejorar la calidad de vida de las localidades. En este sentido se consolida la visión y tratamiento de los problemas sociales desde una perspectiva espacial, con políticas de actuación referidas a ámbitos y colectivos concretos.

- En conjunto, el tipo de prácticas presentadas son de poca envergadura en cuanto al presupuesto y amplitud de la actuación, aunque todas son muy homogéneas en cuanto a la temática a la que iba dirigida, presentan un carácter innovador y tienen un claro interés.

Junto a estos elementos básicos y muy positivos, se puede detectar algunas carencias en la relación de propuestas:

- En primer lugar, la escasa atención que las propuestas realizan a problemas de exclusión social especializados (inmigración, enfermos crónicos, vejez, diferencias étnicas, problemas de viviendas, seguridad ciudadana, prevención de la delincuencia, etc), tan sólo la práctica del municipio de Aserrí y una de Navolato atienden a aspectos de salud. 
- El segundo límite que se puede destacar se refiere a que, sólo en contadas ocasiones (caso de la ciudad de Granada, El Bosque y presumiblemente Oyon) se incluye dentro del programa un plan de difusión de la propuesta llevada a cabo de manera que ésta actividad se convierta en modelo de actuación y permita, de esta forma, el establecimiento de un acervo acumulativo de ideas, recursos, actividades, agentes, etc. En relación a esta carencia el interés del proyecto Urbal por la divulgación de las Buenas Prácticas seleccionadas.

- El tercer elemento se refiere a que muy pocos programas o propuestas (salvo la de Lima, Perú) incluyen un esquema riguroso de evaluación o autoevaluación de las actividades que permitieran criterios de seguimiento, de control y, eventualmente, de corrección de la actuación o actuaciones desarrolladas

- Por último, vuelven a ser pocas las prácticas que utilizan los sistemas de información geográficas, si bien todas ellas en un futuro pueden implementarse a través de esta técnica.

\section{3. Áreas temáticas}

Como es lógico la totalidad de las prácticas tienen como área temática principal situaciones de Exclusión/Vulnerabilidad Social. Pero si en lugar de referirnos tan sólo al área principal a la que se asocia la práctica, consideramos los aspecto específicos a los que atienden cada una de las Buenas Prácticas seleccionadas, hay que calificarlas de diversas. Cada una de las ciudades participantes ha puesto el acento en una vertiente específica de la exclusión social (Cuadro 5).

Las acciones contra la exclusión social afectan a modificaciones de las problemática de los colectivos más vulnerables (niños, adolescentes, jóvenes, etc), a esquemas de la organización comunitaria del ámbito sobre el que estas actividades se llevan a cabo, o a la mejora técnica de los medios de lo/s organismo/s implicados. Específicamente, las distintas prácticas se pueden agrupan en diferentes categorías:

1.- Experiencias de fortalecimiento institucional. Son aquellas experiencias de gestión municipal en las cuales se logran cambios en los métodos convencionales de actuación de la administración, expresándose en el fortalecimiento de las estructuras organizativas, donde se enfatiza la calidad de los resultados y la gestión de los recursos, en la que se cuenta con la participación activa de los funcionarios.

- En esta categoría tendría que estar incluida la mayor parte de las prácticas puesto que uno de los objetivos del proyecto era el desarrollo 
tecnológico de la administración a través de la implementación de un SIG. Si bien este es el objetivo último del proyecto, con lo que cabe esperar que todas las ciudades participantes lo lleve a corto plazo a buen término.

- En el momento de remisión y selección de las acciones sólo las de Granada, Málaga y El Bosque presentan la estructura informática y técnica totalmente acorde con los objetivos. Han sido experiencias que han pretendido la utilización de herramientas y mecanismos que mejorarán y facilitarán la gestión municipal en diversos ámbitos, a nivel educacional (Chile), a nivel de empleo (Granada), en el funcionamiento mismo de los servicios sociales (Málaga).

2.- Experiencias de desarrollo social. Son aquellas experiencias de gestión municipal en las cuales el desarrollo de políticas y estrategias municipales apuntan a generar impacto en la mejora de las condiciones de vida de la ciudadanía en el ámbito social, de educación, de salud y de superación de la pobreza, donde las decisiones sean consistente con los objetivos y problemas detectados, así mismo con las políticas públicas y desarrollo social existentes. Dentro de este ámbito se incluyen la mayoría de las actividades, las cuáles podemos subdividirlas en varias subáreas dependiendo de la problemática específica a la que se dirija:

- Problemáticas que afectan a la población juvenil-adolescente. En este grupo se incluye un conjunto de prácticas cuyo objetivo fundamental es a la vez que atajar la problemática que actualmente cuenta este colectivo de población, prevenir los problemas que a medio y largo plazo podría generar este grupo si no se les atiende de forma adecuada: delincuencia, desectructuración familiar, etc. En líneas generales las principales problemáticas atendidas han sido las situaciones de riesgo social y adicción que sufren los niños y adolescentes, especialmente sensible en aquel colectivo que se encuentra fuera del hogar, que son los llamados "habitantes de la calles", es el caso de los actividades desarrolladas por Navolato y Feira de Santana.

- El género y la vulnerabilidad social, una temática de actualidad y que continuamente aparece en la palestra pública ha sido considerado en una actividad remitida por el municipio de Navolato, en la línea de la violencia intrafamiliar.

- También se contempla la atención a la salud. Ésta ha sido la temática de dos prácticas, una remitida por Aserrí que pretende conseguir un buen servicio de salud integral estableciendo cuáles son los sectores que necesitan mayor concentración de recursos; y otra desarrollada por Navolato que aborda la salud desde una perspectiva psicosocial. 
- Los problemas de empleo han sido tratados por la ciudad de Granada mediante una práctica que intenta mejorar la gestión municipal en esta línea.

3.- Experiencias de desarrollo comunitario en el ámbito urbano. Se trata únicamente de la experiencia enviada por Lima (Perú) que pretende proveer a la comunidad de un modelo de intervención para su desarrollo económico y social.

\section{CUADRO 5 \\ PRINCIPALES TEMÁTICAS DE LAS BUENAS PRÁCTICAS SELECCIONADAS}

\begin{tabular}{|l|c|l|}
\hline PAÍS & N $^{\text {PRACTICAS }}$ & TEMÁTICA \\
\hline ASERRI (COSTA RICA) & 1 & $\bullet$ Gestión de los recursos de salud \\
\hline FEIRA DE SANTANA (BRASIL) & 1 & $\begin{array}{l}\bullet \text { Problemática de niños y } \\
\text { adolescentes }\end{array}$ \\
\hline OYON (PERÚ) & 1 & $\begin{array}{l}\bullet \text { Bases de datos estadísticas para } \\
\text { aplicar a un SIG }\end{array}$ \\
\hline NAVOLATO (MÉJICO) & 3 & $\begin{array}{l}\bullet \text { Conductas adictivas y sus } \\
\text { consecuencias } \\
\bullet \text { Problemáticas juveniles } \\
\bullet \text { Género e inclusión social }\end{array}$ \\
\hline EL BOSQUE (CHILE) & 2 & $\begin{array}{l}\bullet \text { Educación-formación } \\
\bullet \text { Gestión municipal }\end{array}$ \\
\hline GRANADA (ESPAÑA) & 2 & $\begin{array}{l}\bullet \text { Empleo y riesgo de exclusión social } \\
\bullet \text { Modelo sociodemográfico }\end{array}$ \\
\hline MALAGA (ESPAÑA) & 2 & $\begin{array}{l}\bullet \text { Diagnóstico social de zonas } \\
\bullet \text { Estudio socio-territorial }\end{array}$ \\
\hline LIMA (PERÚ) & 1 & $\bullet$ Integración en comunidad \\
\hline
\end{tabular}

\subsection{Organismos participantes}

Los organismos que han enviado la totalidad de las prácticas han sido los Ayuntamientos. Las diferencias entre unos y otros están ligadas a la tipología de las prácticas en función de lo cual dependerá de una instancia u otra dentro de esta institución, de esta forma las prácticas de Málaga son remitidas por el Área de Bienestar Social del Ayuntamiento, las de Granada por el Instituto de Formación y empleo del Ayuntamiento, etc, tal y como se recoge en el cuadro 6.

En cuanto a los organismos participantes, todas las prácticas, a excepción de la enviada por la ciudad de Granada, han cumplido con uno de los requisitos básicos que engloban los criterios de evaluación, la participación de varias institu- 
ciones en su elaboración y puesta en práctica. En todas ellas la instancia ejecutora principal ha sido los ayuntamientos, pero además ha sido frecuente la participación de Delegaciones y Ministerios, ONGs y algunas asociaciones ciudadanas. Es de destacar una Buena Práctica remitida por Aserrí donde la participación de instituciones ha sido bastante cuantitativa, un total de 39 instituciones (Cuadro 7), las de Navolato donde implícitamente participa la UE al ser parte del programa Hábitat, la de Perú donde se ha contabilizado la participación internacional, y la de Málaga donde se ha contado con la participación universitaria.

\section{CUADRO 6 \\ ORGANISMO DE REMISIÓN DE LAS BUENAS PRÁCTICAS SELECCIONADAS}

\begin{tabular}{|c|c|c|}
\hline PAÍS & $\begin{array}{c}\mathrm{N}^{\mathrm{o}} \\
\text { PRACTICAS }\end{array}$ & $\begin{array}{l}\text { ORGANISMOS DE REMISIÓN } \\
\text { DE LA PRÁCTICA }\end{array}$ \\
\hline ASERRI (COSTA RICA) & 1 & - Área rectora de Aserrí \\
\hline FEIRA DE SANTANA (BRASIL) & 1 & - Ayuntamiento de Feira de Santana \\
\hline OYON & 1 & -Municipalidad de Oyon \\
\hline NAVOLATO (MÉJICO) & 3 & - Secretaría de Desarrollo Social \\
\hline EL BOSQUE (CHILE) & 2 & $\begin{array}{l}\text { - Municipalidad de El Bosque. } \\
\text { Unidad de SIG }\end{array}$ \\
\hline GRANADA (ESPA NA $)$ & 2 & $\begin{array}{l}\text { - Instituto de Formación y Empelo } \\
\text { Ayuntamiento de Granada }\end{array}$ \\
\hline MALAGA (ESPAÑA) & 2 & $\begin{array}{l}\text { - Área de Bienestar Social, } \\
\text { Ayuntamiento de Málaga }\end{array}$ \\
\hline LIMA (PERÚ) & 1 & - Gerencia de desarrollo social \\
\hline
\end{tabular}

\section{CUADRO 7}

\section{ORGANISMOS PARTICIPANTES EN LAS BUENAS PRÁCTICAS SELECCIONADAS}

\begin{tabular}{|c|c|c|}
\hline PAÍS & $\begin{array}{c}\mathrm{N}^{\circ} \\
\text { PRACTICAS }\end{array}$ & $\begin{array}{l}\mathrm{N}^{\circ} \text { de ORGANISMOS } \\
\text { PARTICIPANTES }\end{array}$ \\
\hline ASERRI (COSTA RICA) & 1 & $\begin{array}{l}-4 \text { principales } \\
\text {-35 secundarias }\end{array}$ \\
\hline FEIRA DE SANTANA (BRASIL) & 1 & $\bullet 15$ \\
\hline OYON (PERÚ) & 1 & $\bullet 4$ \\
\hline NAVOLATO (MÉJICO) & 3 & - De 2 a 3 \\
\hline EL BOSQUE (CHILE) & 2 & $\bullet 3$ \\
\hline GRANADA (ESPAÑA) & 2 & $\bullet 1$ \\
\hline MALAGA (ESPAÑA) & 2 & $\begin{array}{l}\cdot 3 \\
\bullet 2\end{array}$ \\
\hline LIMA (PERU) & 1 & $\bullet 8$ \\
\hline
\end{tabular}




\subsection{Presupuesto, período de aplicación y población afectada}

Uno de los objetivos de sostenibilidad de las prácticas está relacionado con sus posibilidades económicas de implantación. En esta línea, las prácticas presentadas y seleccionadas en cuanto al presupuesto invertido poseen altas posibilidades de extrapolación a otros territorios, en cuanto que con recursos económicos bastantes moderados han obtenidos excelentes resultados. Una explicación de los parcos presupuestos de las prácticas se debe a que la mayoría de ellas han sido ideadas y puesta en funcionamiento por los funcionarios que ya estaban contratados en las distintas áreas, por lo que el salario de éstos no se contemplan directamente dentro del presupuesto habilitado para la práctica, aunque indirectamente tienen un peso considerable. Algunas prácticas sólo contemplan como coste económico el presupuesto inherente al material técnico para iniciar su funcionamiento, éste es el caso de las prácticas desarrolladas por los municipios de Oyon, Granada, Málaga y Chile, además esta última debido a la larga andadura que tiene los SIG, ha sido muy escaso el material técnico de nueva adquisición. En el presupuesto de la mayoría de las prácticas la partida más considerable va dirigida a la adquisición del material informático y cartográfico para su puesta en marcha.

Como podemos apreciar en el cuadro 8 , los presupuestos han oscilado entre dos extremos bastante amplios, los $189 \$$ de Chile y más de 200000 Euros de las prácticas de Navolato, cuyo presupuesto se dispara respecto a las restantes al formar parte de un proyecto internacional "Proyecto Habitat".

\section{CUADRO 8 \\ PRESUPUESTO DE LAS BUENAS PRÁCTICAS SELECCIONADAS}

\begin{tabular}{|c|c|c|c|c|}
\hline PAÍS & PRÁCTICAS & PRESUPUESTOS & $\begin{array}{c}\text { PERIODO } \\
\text { APLICACIÓN }\end{array}$ & $\begin{array}{c}\text { EDADES } \\
\text { POBLACIÓN }\end{array}$ \\
\hline & 1 & $155.000 \$$ & $1999-2005$ & $\begin{array}{c}\text { Toda la } \\
\text { población }\end{array}$ \\
\hline $\begin{array}{l}\text { FEIRA DE SANTANA } \\
\text { (BRASIL) }\end{array}$ & 1 & ---------- & 2002 & $\begin{array}{c}\text { Niños y } \\
\text { adolescentes }\end{array}$ \\
\hline OYON (PERÚ) & 1 & $\begin{array}{l}5060 \$ \text { (Mapas y } \\
\text { software) }\end{array}$ & $1993-2005$ & $\begin{array}{c}\text { Todas las } \\
\text { edades }\end{array}$ \\
\hline NAVOLATO (MÉJICO) & 3 & $\begin{array}{c}580.800 \$ \\
240000.00 \$ \\
272500.00 \$\end{array}$ & 2004-2005 & $\begin{array}{c}\text { Jóvenes } \\
\text { Adolescentes y } \\
\text { jóvenes } \\
\text { Todas las } \\
\text { edades }\end{array}$ \\
\hline
\end{tabular}




\begin{tabular}{|c|c|c|c|c|}
\hline PAÍS & PRÁCTICAS & PRESUPUESTOS & $\begin{array}{c}\text { PERIODO } \\
\text { APLICACIÓN }\end{array}$ & $\begin{array}{c}\text { EDADES } \\
\text { POBLACIÓN }\end{array}$ \\
\hline EL BOSQUE (CHILE) & 2 & $189 \$$ & $\begin{array}{c}2004 \\
1999-2003\end{array}$ & $\begin{array}{c}\text { Edad escolar } \\
(4-6 \text { años }) \\
\text { Toda la } \\
\text { población }\end{array}$ \\
\hline GRANADA (ESPAÑA) & 2 & ------ & 6 Meses & $\begin{array}{c}\text { Personal técnico } \\
\text { Población 24-64 } \\
\text { años }\end{array}$ \\
\hline MALAGA (ESPAÑA) & 2 & $\begin{array}{c}\text { Salario personal } \\
60.100 \text { Euros }\end{array}$ & $\begin{array}{c}12 \text { Meses } \\
(2005)\end{array}$ & $\begin{array}{c}\text { Toda la } \\
\text { población }\end{array}$ \\
\hline LIMA (PERÚ) & 1 & ------- & 12 Meses & Hogares \\
\hline
\end{tabular}

La mayoría de las prácticas se han aplicado en un período bastante limitado de tiempo, entre seis meses y un año, ello no implica que sus manifestaciones se reduzcan a este tiempo, sino por el contrario que ha sido el período de puesta en práctica siendo su período de andadura y obtención de resultados más amplio. Cuatro de ellas, la de Oyon, una de Navolato, otra de Chile y la de Costa Rica, al ser prácticas que se pusieron en marcha hace más tiempo, aparentemente presentan un período de aplicación mayor entre 3 y seis años (Cuadro 9).

En relación a la población a la que va dirigida lo más frecuente es que las prácticas se dirijan a todo el colectivo de población, aunque cuando se especifica a un grupo de población en concreto éste suele coincidir con la población de menor edad, esto es niños, jóvenes y adolescentes. De esta tendencia general se aparta la práctica remitida por Granada que va dirigida al personal técnico de la administración con el objetivo de facilitar y agilizar su actividad en la detección de los problemas sociales de la ciudad.

\section{COMENTARIOS FINALES}

Como conclusión final podemos resaltar que todas las propuestas consideradas tienen un gran interés por una doble razón:

- En primer lugar, por el impacto y la dinámica que han logrado en los ámbitos donde se han puesto en marcha.

- En segundo lugar, por el carácter de referente de interés para otras comunidades con problemas similares.

Estas experiencias pueden clasificarse en función de su centro de interés prioritario inicial. Así, algunas de ellas focalizan su trabajo en el entorno, su mejora, adecuación, etc. En otros casos, la mayoría, la intervención se articula 
a partir de su interés en las personas (inclusión social, mejora de patologías sociales, adicciones, etc).

Del conjunto de Buenas Prácticas presentadas y seleccionadas se pueden extraer una serie de enseñanzas:

- En primer lugar, se trata de prácticas pioneras puesto que han sido puestas en marcha por primera vez en cada uno de los municipios integrantes del proyecto, lo que indica una gran necesidad de reforzar las redes existentes de intercambio de información y conocimientos.

- En segundo lugar y en paralelo con lo anterior, la existencia de una gran riqueza de esfuerzos, capacidad de actuación, creatividad y nuevas formas de actuación para enfrentarse a los nuevos retos a los que tienen que hacer frente los núcleos urbanos.

- En tercer lugar que existe también una gran demanda de información y de búsqueda de intercambio de experiencias y de información del conocimiento como un instrumento tan importante o más que la capacidad económica para resolver o hacer frente a los problemas.

Por último, hay que señalar que las Buenas Prácticas carecen de sentido y objetivo si no se difunden, en este sentido el Proyecto Urbal incluía dos tipos de actividades para la divulgación, una es la difusión de las Buenas Prácticas mediante la publicación de un Catálogo de las experiencias seleccionadas, y otro la realización de seminarios (Málaga, septiembre de 2005) de presentación de los resultados, con una exposición y presentación sobre las Buenas Prácticas que han presentado cada país miembro.

En última instancia hay que decir que la valoración global del proceso ha sido muy positiva, pero que habría que señalar que no se han cumplido los objetivos iniciales en el ámbito de las nuevas tecnologías de la información, pero que se espera que a corto plazo esta carencia sea subsanada. 


\section{BIBLIOGRAFÍA}

ÁREA RECTORA DE ASERRÍ (1992-2005): Buenas Prácticas llevadas a cabo en Aserrí (Costa Rica)

AYUNTAMIENTO DE FEIRA DE SANTA (2002): Buenas Prácticas llevadas a cabo en Feira de Santana (Brasil)

MUNICIPALIDAD DE OYON (1993-2005): Buenas Prácticas llevadas a cabo en Oyon (Perú)

SECRETARÍA DE DESARROLLO SOCIAL (2004-2005): Buenas Prácticas llevadas a cabo en Navolato (Méjico)

MUNICIPALIDAD DE EL BOSQUE. UNIDAD DE SIG (1999-2005): Buenas Prácticas llevadas a cabo en El Bosque (Chile)

INSTITUTO DE FORMACIÓN Y EMPLEO. AYUNTAMIENTO DE GRANADA (2000): Buenas Prácticas llevadas a cabo en Granada (España)

ÁREA DE BIENESTAR SOCIAL. AYUNTAMIENTO DE MÁLAGA. (2005): Buenas Prácticas llevadas a cabo en Málaga (España)

GERENCIA DE DESARROLLO SOCIAL (2005): Buenas Prácticas llevadas a cabo por Lima (Perú)

GIMENO ULLASTRE, J.A, (Coord) (2004): Exclusión social y estado del bienestar, Ed. Centro de Estudios Ramón Areces y Fundación Luis Vives, Madrid

MINISTERIO DE SANIDAD Y CONSUMO: Manual de Buenas Prácticas para la atención a drogodependientes en centros de emergencia.

www. diba.es/promoció_economica/bones_practiques/guiaes(inyto.htm www.grupogureal.com/es/guia/calidadservicio.htm

http:/habitat.aq.upm.es/eles/abpes_2.htm1

http:/habitat.aq.upm.es/evbpes_1htm1 
